\title{
COMMENTS ON THE CONTINUITY OF DISTRIBUTION FUNCTIONS OBTAINED BY SUPERPOSITION ${ }^{1}$
}

\author{
BARTHEL W. HUFF
}

\begin{abstract}
Let $\{X(t)\}$ be a differential process and $Y$ a nonnegative random variable independent of the process. We consider whether the superposition $X(Y)$ can have a continuous probability distribution. If the process has continuous distributions, then the superposition is continuous if and only if $P[Y=0]=0$. If the process has discontinuous distributions and no trend, then no superposition can have continuous distribution. If the process has discontinuous distributions and nonzero trend, then the superposition onto a random epoch has continuous distribution if and only if $Y$ has continuous distribution.
\end{abstract}

1. Introduction. Our terminology is, in general, that of [4]. Suppose that $Y, X_{1}, X_{2}, \cdots$ are independent random variables with $\mathscr{L}(Y)$ $=\odot(\lambda)$ and that $X_{1}, X_{2}, \cdots$ are identically distributed. Clearly, the distribution function of the random sum $Z=X_{1}+\cdots+X_{Y}$ will have a jump discontinuity of size greater than or equal to $P[Y=0]$ $=e^{-\lambda}$ at the origin. A differential process $\{X(t) / t \in[0, \infty)\}$ is a stochastic process with stationary, independent increments that is continuous in law and satisfies the initial condition $P[X(0)=0]=1$. Note that if $Y$ is independent of $\{X(t)\}$, then the superposition $X(Y)$ is a random sum as described above. We shall consider such superpositions when $Y$ is not necessarily integer-valued and concern ourself with the question of whether or not the superposition has continuous distribution.

Every differential process is an infinitely divisible process; that is, the characteristic functions of the process are of the form

$$
\begin{aligned}
f_{X(t)}(u)=\exp \left\{t \left(i \gamma_{X} u-\sigma_{X}^{2} u^{2} / 2\right.\right. & \\
& \left.\left.\quad+\int_{-\infty}^{\infty}\left(e^{i u x}-1-\frac{i u x}{1+x^{2}}\right) d M_{X}(x)\right)\right\},
\end{aligned}
$$

Received by the editors April 6, 1970.

AMS subject classifications. Primary 6020; Secondary 6040.

Key words and phrases. Superposition, infinitely divisible, Lévy parameters, differential process, random epoch, random sum.

1 This research was supported in part by the National Aeronautics and Space Administration under NASA Grant NGR 11-003-020.

Copyright @ 1971, American Mathematical Society 
where $\gamma_{X}, \sigma_{X}^{2}$, and $M_{X}$ are the Lévy parameters uniquely associated with the given distribution. The Lévy spectral function $M_{X}$ is nondecreasing on $(-\infty, 0)$ and $(0, \infty)$, is asymptotically zero $\left(M_{X}(-\infty)\right.$ $=0=M_{X}(\infty)$ ), and satisfies the integrability condition

$$
\int_{-1}^{-0}+\int_{+0}^{+1} x^{2} d M_{X}(x)<\infty
$$

P. Hartman and A. Wintner in [2] and J. R. Blum and M. Rosenblatt in [1] have proved that a necessary and sufficient condition for an infinitely divisible random variable to have continuous distribution is that $\int_{-\infty}^{\infty} d M(x)=\infty$ or $\sigma^{2}>0$. Consequently, if the $\{X(t)\}$ process has discontinuous distributions, then its characteristic functions can be written in the form

$$
f_{X(t)}(u)=\exp \left\{t\left(i \tau_{X} u+\int_{-\infty}^{\infty}\left(e^{i u x}-1\right) d M_{X}(x)\right)\right\},
$$

where $\tau_{X}$ is the trend term of the process.

If $Y \geqq 0$ is independent of $\{X(t)\}$, then it is well known that the superposition $X(Y)$ has characteristic function

$$
f_{X(Y)}(u)=\int_{-\infty}^{\infty} f_{X(t)}(u) d F_{Y}(t) .
$$

Applying the Helly-Bray Theorem, we see that if $\left\langle Y_{n}\right\rangle$ and $Y$ are independent of $\{X(t)\}$ and $Y_{n} \stackrel{\mathcal{L}}{\rightarrow} Y$, then $X\left(Y_{n}\right) \stackrel{\mathfrak{L}}{\rightarrow} X(Y)$. The Lebesgue Dominated Convergence Theorem implies that if $Y$ is independent of the differential processes $\left\{X_{n}(t)\right\}$ and $X_{n}(t) \stackrel{\mathscr{L}}{\rightarrow} X(t)$, $\forall t$, then $X_{n}(Y) \stackrel{\mathfrak{L}}{\rightarrow} X(Y)$.

\section{Superpositions of processes.}

THEOREM 1. Let $\{X(t) / t \in[0, \infty)\}$ be a differential process and let $Y$ be a nonnegative random variable that is independent of the process. The distribution function of the superposition $X(Y)$ is given by

$$
\begin{aligned}
F_{X(Y)}(x) & =P[Y=0]+\int_{0}^{\infty} F_{X(t)}(x) d F_{Y}(t), & & x \geqq 0, \\
& =\int_{0}^{\infty} F_{X(t)}(x) d F_{Y}(t), & & x<0,
\end{aligned}
$$

at points of continuity. 
The proof is sketched for $x \geqq 0$ fixed. We introduce auxiliary processes $\left\{Z_{n}(t)\right\}$ to avoid the complication caused by the fact that the stochastic distribution function $F_{X(t)}(x)$ may not be continuous as a function of $t$. For each positive integer $n$ let $\left\{Z_{n}(t)\right\}$ be a differential process, independent of $\{X(t)\}$ and $Y$, such that $Z_{n}(t)$ is a nonpositive random variable with characteristic function

$$
f_{Z_{n}(t)}(u)=\exp \left\{\frac{t}{n} \int_{-\infty}^{0}\left(e^{i u x}-1\right) d_{x}\left(1 /|x|^{1 / 2}\right)\right\} .
$$

(Such processes can be generated via the first exit times of a Wiener process.) Since the spectral function assigns infinite mass to $(-\infty, 0)$, we know that $Z_{n}(t)$ has continuous distribution. The Central Limit Theorem and the nonpositiveness guarantee that $F_{Z_{n}(t)}(\cdot)$ converges everywhere to the distribution with all mass at the origin as $n$ tends to $\infty$. Now consider the infinitely divisible processes $\left\{X_{n}(t)=X(t)\right.$ $\left.+Z_{n}(t)\right\}$. By the Lebesgue Dominated Convergence Theorem

$$
\begin{aligned}
F_{X_{n}(t)}(x) & =\int_{-\infty}^{\infty} F_{Z_{n}(t)}(x-y) d F_{X(t)}(y) \\
& \rightarrow \int_{-\infty}^{x} d F_{X(t)}(y)=F_{X(t)}(x), \quad \forall x .
\end{aligned}
$$

Moreover, $\left\{X_{n}(t)\right\}$ is continuous in law and each random variable $X_{n}(t)$ has continuous distribution. Thus $F_{X_{n}(t)}(x)$ is continuous as a function of $t$.

Now set

$$
Y_{m}=0 \cdot I_{[Y=0]}+\sum_{k=1}^{\infty} \frac{k}{2^{m}} \cdot I_{\left[(k-1) / 2^{m}<Y \leq k / 2^{m}\right]}
$$

Then

$$
\begin{aligned}
F_{X_{n}\left(Y_{m}\right)}(x) & =P\left[X_{n}\left(Y_{m}\right) \leqq x\right] \\
& =P[Y=0]+\sum_{k=1}^{\infty} P\left[X_{n}\left(k / 2^{m}\right) \leqq x\right] \cdot P\left[Y_{m}=k / 2^{m}\right] \\
& =P[Y=0]+\sum_{k=1}^{\infty} F_{X_{n}\left(k / 2^{m}\right)}(x) \cdot P\left[Y_{m}=k / 2^{m}\right] \\
& =P[Y=0]+\int_{0}^{\infty} F_{X_{n}(t)}(x) d F_{Y_{m}}(t) .
\end{aligned}
$$


Now $Y_{m} \downarrow Y$ so $F_{Y_{m} \stackrel{c}{\rightarrow}} F_{Y}$. Thus the Helly-Bray Theorem yields

$$
F_{X_{n}(Y)}(x)=P[Y=0]+\int_{0}^{\infty} F_{X_{n}(t)}(x) d F_{Y}(t) .
$$

An application of the Lebesgue Dominated Convergence Theorem completes our proof.

Applying the Lebesgue Dominated Convergence Theorem to (A), we obtain

Corollary 1A. Let $\{X(t)\}$ be a differential process with continuous distributions and let $Y$ be a nonnegative random variable that is independent of the process with $P[Y=0]=\gamma$. Then the probability distribution function of the superposition is continuous except for a jump of size $\gamma$ at the origin.

\section{Superpositions of processes with discontinuous distributions.}

Theorem 2. Let $\{X(t)\}$ be any differential process with discontinuous distributions and zero trend term. Then no superposition can have continuous distribution.

PRoof. We shall show that such a superposition must have a discontinuity at the origin. (A) clearly implies such a discontinuity if $P[Y=0]>0$ so, without loss of generality, we assume $Y>0$. If the process has discontinuous distributions, then we know that $\int_{-\infty}^{\infty} d M_{X}(x)<\infty$. Let $\mu=\int_{-\infty}^{0} d M_{X}(x)$ and $\lambda=\int_{0}^{\infty} d M_{X}(x)$. We may assume that $\mu+\lambda>0$ since the claimed result is trivial if $X(t) \equiv 0, \forall t$. If we define a distribution function $G$ via

$$
\begin{aligned}
G(x) & =M_{X}(x) / \mu+\lambda, & & x<0, \\
& =\mu / \mu+\lambda, & & x=0, \\
& =\left(\mu+\lambda+M_{X}(x)\right) / \mu+\lambda, & & x>0,
\end{aligned}
$$

then $F_{X(t)}(\cdot)$ is the distribution function of the random sum $Z=X_{1}$ $+\cdots+X_{Y}$, where $X_{1}, X_{2}, \cdots$ are independent with common distribution $G$ and $\mathscr{L}(Y)=\mathcal{P}(t(\mu+\lambda))$. This follows from Theorem 6 of $\S 6.1$ in [4]. Indeed if $g(u)$ is the characteristic function of $X_{1}$, then

$$
\begin{aligned}
f_{Z}(u) & =\exp \{t(\mu+\lambda)[g(u)-1]\} \\
& =\exp \left\{t(\mu+\lambda) \int_{-\infty}^{\infty}\left(e^{i u x}-1\right) d G(x)\right\} \\
& =\exp \left\{t \int_{-\infty}^{\infty}\left(e^{i u x}-1\right) d M_{X}(x)\right\} .
\end{aligned}
$$


It is now clear that $F_{X(t)}(\cdot)$ has a jump at the origin of magnitude at least $e^{-t(\mu+\lambda)}$. Using $(\mathrm{A})$ and the Lebesgue Dominated Convergence Theorem, we see that

$$
F_{X(Y)}(0)-F_{X(Y)}(0-) \geqq \int_{0}^{\infty} e^{-t(\mu+\lambda)} d F_{Y}(t)>0
$$

and the superposition has a discontinuity at the origin.

The preceding result says that a differential process with discontinuous distributions and no trend or drift is in some sense "bad" and the discontinuities cannot be smoothed out by means of a superposition. The presence of a trend term, however, insures that some superpositions must be continuous. In particular, suppose that $Y$ is a random epoch; i.e., a nonnegative infinitely divisible random variable with spectral function satisfying $\int_{+0}^{+1} x d M_{Y}(x)<\infty$. The question of whether or not the superposition has continuous distribution can be answered by

Theorem 3. Let $\{X(t)\}$ be a differential process with discontinuous distributions and nonzero trend term. Let $Y$ be any independent random epoch. Then the superposition $X(Y)$ has continuous distribution if and only if $Y$ has continuous distribution.

Proof. V. M. Zolotarev in [5] and the author in [3] have shown that the superposition is an infinitely divisible random variable with no gaussian component and Lévy spectral function

$$
M^{*}(x)=\tau_{Y} M_{X}(x)+\int_{0}^{\infty} F_{X(t)}^{*}(x) d M_{Y}(t),
$$

where the stochastic distribution function is defined via

$$
\begin{aligned}
F_{X(t)}^{*}(x) & =F_{X(t)}(x), & & x<0, \\
& =F_{X(t)}(x)-1, & & x>0 .
\end{aligned}
$$

We note that the superposition has continuous distribution if and only if $\int_{-\infty}^{\infty} d M^{*}(x)=\infty$. It is clear that this cannot happen if $\int_{0}^{\infty} d M_{Y}(t)$ $<\infty$ and the necessity of $Y$ having continuous distribution is easily established.

Now suppose that $Y$ has continuous distribution and that the differential process has positive trend. We shall show that the spectral function of the superposition must assign infinite mass to the positive half-axis. Using the argument and notation of Theorem 2, 
we see that $F_{X(t)}(\cdot)$ takes a jump of magnitude at least $e^{-t(\mu+\lambda)}$ at $t \tau_{X}$. Thus for $x>0$, the superposition has spectral function

$$
\begin{aligned}
M^{*}(x) & =\int_{0}^{\infty} F_{X(t)}^{*}(x) d M_{Y}(t) \\
& \leqq \int_{x / \tau}^{\infty} F_{X(t)}^{*}(x) d M_{Y}(t) \\
& \leqq-\int_{x / \tau_{X}}^{\infty} e^{-t(\mu+\lambda)} d M_{Y}(t) .
\end{aligned}
$$

The Lebesgue Monotone Convergence Theorem now implies that $M^{*}(0+)=-\infty$. and the superposition has continuous distribution. A similar argument for a differential process with negative trend completes the proof.

It is not clear that Theorem 3 would be valid if $Y$ were not a random epoch. The technique of the proof obviously cannot be extended.

\section{REFERENCES}

1. J. R. Blum and M. Rosenblatt, On the structure of infinitely divisible distributions, Pacific J. Math. 9 (1959), 1-7. MR 21 \#4465.

2. P. Hartman and A. Wintner, On the infinitesimal generators of integral convolutions, Amer. J. Math 64 (1942), 273-298. MR 4, 18.

3. B. W. Huff, The strict subordination of differential processes, Sankyā Ser. A 31 (1969), 403-412.

4. H. G. Tucker, A graduate course in probability, Probability and Math. Statist., vol. 2, Academic Press, New York, 1967. MR 36 \#4593.

5. V. M. Zolotarev, Distribution of the superposition of infinitely divisible processes. Teor. Verojatnost. i Primenen. 3 (1958), 197-200=Theor. Probability Appl. 3 (1958), 185-188. MR 23 \#A683.

University of Georgia, Athens, Georgia 30601 